\title{
Representação Mental e Mudança Terapêutica: Uma Contribuição da Perspectiva Psicanalítica da Teoria das Relações Objetais
}

\author{
Denise Zanatta \\ Silvia Pereira da Cruz Benetti ${ }^{1}$ \\ Universidade do Vale do Rio dos Sinos, UNISINOS
}

\begin{abstract}
RESUMO - Apesar do avanço das pesquisas em psicoterapia, estudos na abordagem psicanalítica ainda são escassos. O objetivo deste artigo é apresentar e discutir a contribuição do conceito de representação mental como um constructo importante para fundamentar pesquisas na área psicanalítica baseadas na identificação dos elementos associados à mudança terapêutica. Inicialmente discutem-se conceitos e modelos teóricos psicanalíticos para posteriormente discutir a noção de representação mental em relação à mudança terapêutica, destacando-se a contribuição da teoria das relações objetais e do apego. Nesse sentido, apresenta-se o Inventário das Relações de Objeto, instrumento que avalia a qualidade das representações mentais, identificando mudanças ao longo da psicoterapia. Espera-se oferecer subsídios para o aprimoramento do trabalho clínico em psicoterapias e o desenvolvimento de pesquisas em psicanálise.
\end{abstract}

Palavras-chave: Representação mental; mudança terapêutica; psicoterapia psicanalítica; relações objetais.

\section{Mental Representation and Therapeutic Change: A Contribution from the Psychoanalytical Approach of Object Relations Theory}

\begin{abstract}
In spite of the progress realized in psychotherapeutic research, studies in the psychoanalytical approach are still scarce. The objective of this article is to present and to discuss the contribution of the concept of mental representation as an important construct to substantiate psychoanalytic research based on the identification of the elements associated with therapeutic change. First, concepts and psychoanalytic models are presented and after that the notion of mental representation is specifically discussed in relation to therapeutic change, particularly the object relation theory. Thus, the Object Relation Inventory is presented, an instrument designed to assess the quality of mental representations, evaluating changes along psychotherapy. Elements to improve clinical work and the development of research in psychoanalysis are presented.
\end{abstract}

Keywords: mental representation; therapeutic change; psychoanalytic psychotherapy; object relations.

Nas últimas décadas, observa-se que os objetivos das pesquisas em psicoterapia já não se limitam somente na comprovação da eficácia dos tratamentos nos diferentes quadros clínicos, mas dirigem-se para a compreensão dos processos envolvidos na mudança terapêutica. Neste sentido, Russel e Orlinsky (1996) assinalam que os estudos em psicoterapia não se restringem ao questionamento da efetividade destas intervenções e, sim, voltam-se para a compreensão de etapas mais específicas do tratamento, tais como a identificação dos processos terapêuticos nas diferentes modalidades de intervenção. Entretanto, apesar do avanço das pesquisas em psicoterapia, estudos embasados na abordagem psicodinâmica são ainda escassos, indicando a necessidade do desenvolvimento de pesquisas sob esta orientação (Zaslavsky, 2005).

Refletindo sobre o menor número de trabalhos com orientação psicanalítica voltados especificamente para pesquisas, Blatt e Auerbach (2003) destacam um aspecto importante do processo terapêutico nesta abordagem. Os autores referem que os resultados esperados no tratamento psicoterápico psicanalítico refletem modificações que vão além da remissão sintomática, indicando câmbios associados às estruturas da personalidade. Neste sentido, o objetivo geral das abordagens

1 Endereço para correspondência: UNISINOS, Av. Unisinos, 950, Bairro Cristo Rei. São Leopoldo, RS. CEP 93022-000.

E-mail: spcbenetti@gmail.com psicanalíticas consiste na mudança das estruturas mentais e dos estados inconscientes, em oposição à grande maioria das pesquisas em psicoterapia, as quais se limitam à análise da redução sintomática.

Além desse aspecto, estudos de revisão sobre a eficácia terapêutica indicam que o melhor aproveitamento terapêutico e maior bem-estar têm sido mencionados pelos pacientes nas situações em que os ganhos da psicoterapia se associaram às mudanças nas formas de lidar com situações difíceis, e não simplesmente com ganhos de redução de sintomática (Blatt, Besser \& Ford, 2007, Blatt \& Auerbach, 2003, Diamond, Blatt, Stayner \& Kaslow, 1991). Logo, o menor número de publicações psicanalíticas não reflete um menor interesse em estudos de eficácia, mas uma posição essencial ao método psicanalítico em relação à mudança terapêutica, orientada pelo propósito de analisar não somente a redução sintomática e, sim, o processo do tratamento.

Avaliações terapêuticas desta ordem aludem a modificações em aspectos representacionais do indivíduo sobre sua situação pessoal e suas relações nos diferentes contextos de vida. Deste modo, constituem-se como transformações associadas a estados representativos e subjetivos do self e a padrões interacionais e afetivos com objetos externos, ambos os conceitos associados ao de representação mental. Assim sendo, partindo da perspectiva das relações objetais Blatt e 
Auerbach (2003) consideram que o conceito de representação mental constitui-se como um referencial importante para o acompanhamento, compreensão e avaliação do processo terapêutico. Portanto, tendo em mente as implicações e as especificidades da mudança terapêutica na perspectiva psicanalítica, pesquisas em psicoterapia investigando conceitos representacionais e subjetivos relativos ao self e a padrões interacionais e afetivos com objetos externos têm sido desenvolvidas focalizando as mudanças terapêuticas (Blatt, Auerbach, Zuroff, \& Shahar, 2006, Blatt, Auerbach \& Levy, 1997, Diamond et al., 1991). Estes trabalhos têm contribuído para a consolidação das investigações em psicanálise e para o desenvolvimento de estratégias de avaliação terapêutica que possibilitam o aprimoramento do trabalho clínico.

A partir destes aspectos, o objetivo deste artigo é discutir as implicações teóricas do conceito de representação mental para a compreensão e identificação dos elementos envolvidos no processo terapêutico e também destacar os fatores associados à mudança terapêutica na perspectiva da psicoterapia de orientação psicanalítica. Inicialmente, iremos abordar alguns conceitos da psicoterapia de orientação psicanalítica e modelos de mudança terapêutica para posteriormente focalizar e discutir especificamente a noção de representação mental em relação ao processo psicoterápico.

\section{Mudança terapêutica e a Psicoterapia de Orientação Psicanalítica}

Na última década, apesar do menor número de trabalhos, observa-se um crescente avanço do número de pesquisas sobre o processo psicoterápico, indicando resultados significativos associados às mudanças terapêuticas de melhora, tanto em psicanálise como em psicoterapia de orientação psicanalítica (Fonagy, Rotha, \& Higgitt, 2005). Em uma revisão da história recente e das perspectivas atuais da pesquisa em psicoterapia de orientação psicanalítica de longa duração em adultos, Jung, Fillippon, Nunes e Eizirik (2006) apontam bons resultados no tratamento do transtorno ansioso, depressivo e de personalidade. Da mesma forma, em relação à infância e adolescência, estudos como os de Kronmuller et al. (2005) e Barish (2004) também indicam resultados importantes de mudança terapêutica em atendimentos psicanalíticos de crianças e adolescentes.

Fundamentalmente, os elementos que sustentam a psicoterapia psicanalítica caracterizam-se pelo uso das bases técnicas da psicanálise - a interpretação, a análise da transferência e a neutralidade técnica do terapeuta (Kernberg, 2003). Técnicas relacionadas à interpretação do material analítico estabelecerão diferenças nas características de três abordagens: psicanalítica clássica, psicoterapia de orientação analítica e psicoterapia psicanalítica de apoio. Enquanto, na primeira abordagem, a psicanálise clássica, a análise transferencial e a interpretação do material se constituem como focos principais do trabalho, na psicoterapia de orientação analítica, estes aspectos são trabalhados com diferenças qualitativas ao longo do tratamento. Isto é, assumem um caráter mais voltado para as situações imediatas de conflito. Desta forma, considera-se que o objetivo da psicanálise é a mudança estrutural, ao passo que, na psicoterapia psicanalítica o objetivo é a reorganização parcial da estrutura psíquica e mudança sintomática (Kernberg, 2003, p. 29). Não obstante, ainda que se destaquem as diferenças nas abordagens, Kernberg aponta que mudanças fundamentais nos indivíduos ocorrem independentes das modalidades, acrescentando que a mudança terapêutica resulta da técnica, da resposta do paciente e do próprio terapeuta.

Pode-se, portanto, considerar que a psicoterapia de orientação psicanalítica tem como objetivo fundamental promover a integração entre o mundo interno e externo do paciente. Sinais de que o processo de mudança está ocorrendo manifestam-se quando o ego se sobrepõe ao id, a estagnação dá espaço para a criatividade e os objetos totais estão no lugar de objetos parciais (Berti, 2004). Todavia, para um maior aprofundamento da compreensão dos processos associados à mudança, também é importante que se considere os diferentes modelos teóricos em psicanálise, os quais contribuem com elementos importantes sobre os aspectos subjetivos do processo terapêutico.

Pine (1998) considera que a psicanálise construiu elementos teóricos importantes sobre o processo terapêutico, a partir de quatro psicologias: psicologia das pulsões, psicologia do ego, psicologia do self e psicologia das relações objetais. $\mathrm{Na}$ psicologia das pulsões, a vida psíquica é compreendida como organizada em torno do conflito e de sua resolução, envolvendo, com isso, a ansiedade, a vergonha, a culpa, a formação de sintomas e os traços de caráter patológicos. As intervenções verbais têm o intuito de produzir gradualmente modificações no conflito, no superego e nos modos inflexíveis de defesa, levando o indivíduo a uma maior aceitação de seus pensamentos e necessidades, à resolução do conflito e a maior tolerância dos afetos. No encontro terapêutico, o analista funciona como um modelo de identificação para o paciente, sendo que a mudança terapêutica se define pela máxima "tornar o inconsciente consciente". Já na perspectiva da psicologia do ego, o indivíduo é visto em termos de sua capacidade de adaptação, teste de realidade e defesas para lidar com o mundo interno de urgências, com os afetos e as fantasias e com o mundo externo das demandas da realidade. A função do terapeuta será, então, descrever, explicar as evoluções e reconstruir as origens das falhas do ego. $\mathrm{Na}$ relação terapêutica, a partir do grau de regressão que foi auxiliado a alcançar pela análise, o paciente começa a colocar em palavras suas experiências, atingindo graus mais altos de desenvolvimento do ego.

A contribuição da psicologia do self reside na ênfase nos estados subjetivos do indivíduo, principalmente em torno de questões relativas aos limites de si e do outro, de continuidade e estima. Os estados de deficiência na experiência do self dizem respeito à baixa estima, às fronteiras frágeis e à descontinuidade. O objetivo, então, é auxiliar o paciente a se familiarizar com seus estados internos para que estes cheguem ao nível da verbalização. Por último, na perspectiva da psicologia das relações objetais, o indivíduo é compreendido em termos de um drama interno, ocorrido na infância precoce e formado pelas experiências com os objetos primários e repetido nas relações atuais. A função do terapeuta é liberar o paciente para vivenciar cada experiência como realmente nova e não absorvê-la no antigo drama de suas relações de objeto internalizadas. 
Na verdade, a ênfase dos modelos corresponde a aspectos do funcionamento psíquico, os quais, por sua vez, convergem para conceitos fundamentais do desenvolvimento psíquico comuns a teoria psicanalítica. Isto é, refletem mudanças das representações internas relativas às estruturas mentais e aos estados inconscientes associados (Blatt \& Auerbach, 2003). Logo, independentemente do foco de cada escola, os efeitos terapêuticos na psicanálise são associados a mudanças básicas na estrutura da personalidade, incluindo neste processo a noção subjacente de representação mental. Ainda que derivada da teoria das relações objetais, a noção de representação mental é uma noção subjacente a toda a compreensão psicanalítica, constituindo-se desta maneira como uma referência fundamental para a compreensão e avaliação do processo terapêutico.

\section{Representação Mental}

O conceito de representação mental, já em 1970, havia se tornado um importante constructo nos estudos psicanalíticos, ainda que associado a diferentes denominações que variavam entre representação da palavra ou coisa, representação do self, representação do objeto, constância objetal, objeto introjetado, dentre outros (Beres \& Joseph, 1970, p. 1).

Todavia, a definição de Novey em 1958, delimitou o conceito de representação mental em relação aos processos perceptivos e os mecanismos de introjeção, projeção e identificação. Desta forma, segundo Beres e Joseph, Novey sintetizou a definição básica do conceito como a representação psicológica dos objetos internos e externos na mente. Ainda assim, os autores destacam que é necessário agregar à conceituação de Novey (1958) o aspecto inconsciente dessas representações. E, neste sentido, consideram que a representação mental dos objetos constitui-se como uma noção fundamental para a compreensão do desenvolvimento do aparelho psíquico, sendo, portanto, a base para toda a atividade psíquica. Na visão psicanalítica do desenvolvimento, a construção das representações mentais, bem como a manutenção e a transformação destas representações são aspectos fundamentais nas teorias das relações objetais e do apego, constituindo uma importante interface entre aspectos da cognição social e do afeto (Ramires, 2003).

Pesquisas sobre a representação mental originam-se fundamentalmente da teoria das relações objetais que considera a qualidade das interações precoces entre a criança e os cuidadores como bases para o desenvolvimento emocional. Estudos desta ordem também são complementados pela perspectiva teórica do apego que igualmente investiga a qualidade dos vínculos iniciais para o estabelecimento dos padrões de apego no indivíduo, os quais determinarão características relacionais posteriores (Diamond \& Blatt, 1994; Blatt \& Levy, 2003). Portanto, ambas as abordagens consideram o desenvolvimento psicológico a partir do papel da matriz relacional do indivíduo.

Partindo da noção freudiana sobre o papel dos impulsos libidinais e agressivos na constituição psíquica do sujeito, a teoria das relações objetais destaca a importância que as primitivas relações diádicas entre a criança e o cuidador desempenham no desenvolvimento das estruturas psíquicas
(Diamond \& Blatt, 1994). Estas experiências interativas são internalizadas e organizadas como uma representação mental do objeto, passando a formar os blocos constituintes das estruturas psicológicas e servindo para orientar o comportamento e a motivação do sujeito. Consequentemente, estas unidades incluem as representações das interações do self e o objeto, o afeto correspondente a esta interação e a representação específica do outro. Estas unidades, self, afeto e objeto, são denominadas relações objetais diádicas (Kernberg, 2005).

Portanto, a representação mental é um construto caracterizado pela capacidade da criança de passar de experiências sensório-motoras para experiências emocionais e afetivas, originando esquemas mentais e descobrindo a sua própria interioridade. Isto é alcançado através do objeto materno, que ao retirar a carga ameaçadora ou insuportável da realidade funciona como um objeto de sustentação e significação, permitindo à criança vivenciar diversificadas experiências. Por sua vez, estas experiências permitem que a criança opere com os objetos e construa representações intrapsíquicas a partir destes. Desta forma, os objetos são substituídos por símbolos, por aquilo que representam a partir de uma experiência emocional única (Matos, 2004).

Em suma, as representações mentais são construídas através de trocas seguras, significativas e emocionais entre a criança e a mãe, com irmãos, pares e, principalmente, com os pais, sendo facilitadas no cenário de brincadeira. Logo, construídas desde as primeiras interações com os cuidadores, resultando em esquemas mentais representativos do self e do objeto que se desenvolvem no ciclo vital. Os elementos que compõem esses esquemas são conscientes e inconscientes, referindo-se a estados afetivos, cognitivos e experienciais. Além disto, refletem o nível de desenvolvimento individual e aspectos do funcionamento psíquico, como impulsos e fantasias (Fonagy \& Target, 1997). Estes esquemas servirão de base para a regulação emocional e comportamental, principalmente nas relações interpessoais, sendo modelo para a maneira como o indivíduo representa a si mesmo e os demais (Blatt et al., 1997).

Por sua vez, a contribuição da teoria do apego para o conceito de representação mental, segundo Diamond e Blatt (1994), centra-se na proposta de Bowlby (1969) acerca do modelo de funcionamento interno (Dalbem \& Dell Áglio, 2006), padrão representacional construído a partir das experiências de apego iniciais. Esta noção se refere ao conjunto das representações internas dos vínculos afetivos estabelecidos com as figuras cuidadoras nas interações iniciais da criança, refletindo também estados internos e do ambiente.

As pesquisas sobre o apego iniciaram-se a partir do trabalho de Bowlby (1969) e Ainsworth (1969) que denominou a ligação entre o bebê e a figura materna como vínculo de apego. Esta ligação não estaria somente determinada por aspectos de dependência física e psicológica do bebê em relação à figura materna, mas também por padrões comportamentais de origem biológica, estabelecidos num sistema de ações voltadas para a manutenção da proximidade entre a criança e sua mãe e consequente formação de vínculo afetivo. Além do caráter de manutenção da proximidade da figura materna, o processo de formação de apego também está relacionado ao desenvolvimento sócio emocional da criança. Através do 
estabelecimento de vínculos de apego também estariam sendo desenvolvidas as representações internas da criança sobre si mesma, sobre os relacionamentos com os demais, bem como as formas de interações e padrões representacionais com efeito duradouro no desenvolvimento individual. O êxito da criança em desenvolver uma representação de apego segura está relacionado a dois fatores fundamentais- ao volume de experiências de estresse vividas e à disponibilidade da figura materna em promover a redução destas experiências frustrantes.

Tanto situações de muito estresse no contexto de desenvolvimento da criança como de não disponibilidade da figura materna estariam relacionadas ao desenvolvimento de padrões de apego não seguros e ao estabelecimento de modelos internos de funcionamento representativos do ambiente e do próprio self da criança como perigoso, inseguro e pouco confiável. Neste sentido, através do trabalho de Ainsworth sobre a teoria do apego foi possível se estabelecer três grandes grupos de estilos de interação que correspondem às classificações de apego seguro, apego inseguro/evitativo e inseguro/ resistente. A cada grupo correspondem uma dinâmica entre a frequência de comportamentos exploratórios, de manifestação de preocupação/ansiedade, e afiliação/habilidade de ser confortado. Posteriormente, foi incluída a classificação apego desorganizado, que corresponderia às características do vínculo estabelecido entre crianças e suas mães em situação de maus-tratos, violência e abuso (Main, 1996).

Diamond e Blatt (1994) consideram que ambas as abordagens, a perspectiva psicanalítica das relações objetais e a teoria do apego, convergem para um entendimento dinâmico e transformativo ao longo do desenvolvimento do processo de elaboração da representação mental, que inclui a representação internalizada do self e do objeto externo. Estas imagos servirão de modelos para interações futuras e organizarão as representações internas do indivíduo, regulando estados afetivos frente às novas experiências ao longo do desenvolvimento.

Pesquisas sobre o processo terapêutico e a representação mental também têm sido desenvolvidas por Fonagy e Target, que trabalham com o conceito de função reflexiva, um construto similar à representação mental e, ao mesmo tempo, complementar a ela, uma vez que a forma como a pessoa representa a si mesma, assim como a possibilidade de mudança terapêutica nesta representação, refletem o desenvolvimento da capacidade reflexiva do sujeito. A função reflexiva consiste na capacidade de reconhecer tanto os estados mentais internos (pensamentos, sentimentos, crença e desejos) como os estados dos demais, dando significado ao comportamento do outro, portanto, distinguindo a realidade interna e externa.

Dessa forma, as representações mentais se transformam ao longo dos anos pelo próprio processo de amadurecimento, tornando-se mais maduras e flexíveis, com capacidade de regular, organizar e dar coerência ao comportamento e estado afetivo interno do indivíduo. Nesse sentido, novas estruturas representacionais se unem em uma interação facilitadora mútua entre o desenvolvimento de um sentido cada vez mais coeso e essencialmente positivo do self e a harmoniosa relação com o objeto, cada vez mais recíproca e empática (Diamond et al., 1991). Ao longo do desenvolvimento individual, as representações mentais, as representações do objeto e do self se tornam cada vez mais diferenciadas, integradas e exatas. Fundamentalmente, estas representações passam a formar esquemas mentais que organizam e regulam o comportamento, o afeto e os padrões interativos do sujeito.

Assim, formas prematuras de representação estão baseadas primariamente em seqüências comportamentais, associadas com necessidades de gratificação, tais como as representações do bebê em relação ao objeto materno. Conforme o desenrolar do desenvolvimento psíquico na infância, organizam-se formas intermediárias, baseadas em características de percepção específicas dos objetos, ligadas essencialmente a aspectos descritivos ou funcionais de representação características do pensamento concreto da criança. As formas mais altas de representação de objeto são mais simbólicas e conceituais e correspondem a etapas maturativas posteriores do indivíduo. Nessa direção, a partir da adolescência, estabelece-se um sentido de si mesmo (self) mais coeso e integrado, permitindo o estabelecimento de uma diferenciação mais precisa em relação aos objetos externos. Ao mesmo tempo em que essas fronteiras se delimitam de forma mais clara, o reconhecimento dos estados internos é também acompanhado pela capacidade de percepção dos estados internos dos demais, assim como seus desejos e afetos. Esses vários níveis de representação, inicialmente originados nas várias fases do relacionamento progenitor(a) - filho(a), tornam-se mais diferenciados, integrados e maduros, determinando a capacidade de estabelecimento de experiências interpessoais satisfatórias, de uma definição do self e de uma identidade mais madura. Consolida-se, então, a capacidade reflexiva, que, além de ser uma habilidade cognitiva, também é uma aquisição psíquica importante, indicativa da complexidade do funcionamento psíquico (Blatt, Chevron, Quinllan, Schaffer \& Wein, 1988)

Com base nesses aspectos, Blatt e Auerbach (2003) assinalam que alguns pontos são centrais no processo de desenvolvimento das representações mentais (representação de si mesmo e do objeto), estabelecendo-se como aspectos centrais ao construto e relacionados às manifestações de psicopatologia. Esses pontos incluem a capacidade do sujeito de estabelecer representações mentais caracterizadas por (a) constância de limites, quando o sujeito estabelece e mantém um senso de separação entre o self e os demais e, internamente, entre o self e o não self; (b) constância emocional, quando o sujeito consegue manter um vínculo com uma pessoa em particular; (c) constância objetal, quando há capacidade de manter uma relação positiva mesmo quando o objeto não está presente, (d) a constância do self, quando o sujeito desenvolve uma representação de si mesmo consolidada, coesa e estável, como diferente e distinta dos demais, ao longo do espaço e do tempo, independentemente do estado emocional e, por último, (e) pensamento operacional, quando se organiza a capacidade de coordenar relações entre diversas dimensões, considerando a si mesmo a partir da perspectiva triádica no âmbito familiar e igualmente em contextos mais amplos, sociais e culturais.

Analisados em conjunto, estes pontos formam a base para a compreensão de diversas manifestações de psicopatologia (Blatt et al., 1997), incluindo, por exemplo, patologias psicóticas, as quais se caracterizam por falhas básicas na capacidade de estabelecimento de limites e no estabelecimento 
da constância objetal. Ou, em quadros de personalidade borderline, quando a constância do self é altamente comprometida pela instabilidade afetiva. Portanto, a análise dos diferentes aspectos associados ao desenvolvimento psíquico e as representações mentais dos objetos podem ser elementos importantes na compreensão dos processos terapêuticos de orientação psicanalítica, bem como na avaliação da mudança terapêutica.

\section{Mudança Terapêutica e Representação Mental}

A partir da compreensão dos aspectos conceituais envolvidos na noção de representação mental, é possível avaliar as características psicológicas dos sujeitos, identificando tanto os níveis representacionais como também mudanças nestes níveis resultantes do processo terapêutico. Neste sentido, a perspectiva terapêutica associada à representação mental sugere que distorções tanto nas representações do self como do objeto podem ser revisadas e mais integradas, a partir de experiências construtivas e positivas no relacionamento terapêutico. Este deve permitir a reformulação de representações distorcidas, que devem ser tornar-se mais integradas, de forma que a mudança terapêutica resultante se baseie em transformações na capacidade reflexiva e habilidades de conexão afetiva e relações interpessoais. Desta forma, no processo terapêutico, espera-se que o trabalho interpretativo sustentado nas vivências transferências possibilite a gradual modificação das representações mentais do self e do objeto, no sentido de uma maior integração e síntese dessas representações, ao nível representativo e afetivo. Justamente, a possibilidade de avaliar essas mudanças é um recurso interessante para o desenvolvimento de pesquisas que avaliem as características da representação mental ao longo do tratamento, verificando as possíveis transformações.

Diversas formas de avaliação da capacidade de representação mental são mencionadas na literatura, incluindo os testes projetivos de Rorschach e TAT (Blatt et al., 2007). Em geral, a teoria e a pesquisa clínica têm focalizado de modo mais significativo na qualidade das primeiras interações do sujeito com os cuidadores e o desenvolvimento das representações do self e do objeto, uma vez que estas organizam as experiências interpessoais subseqüentes (Diamond et al., 1991).

Além dessas técnicas, Gruen e Blatt (1990) desenvolveram um procedimento de análise da representação mental baseado na avaliação de descrições espontâneas de si mesmo (self) e de pessoas significativas, considerando a estrutura e o conteúdo destas verbalizações. Assim, através de uma entrevista semi-estruturada na qual é solicitado ao sujeito descrever a si próprio e também os indivíduos significativos - geralmente mãe, pai- são caracterizadas as representação de si mesmo (self) e as representações objetais do paciente. Utilizando-se de conceitos do desenvolvimento sob a perspectiva psicanalítica e cognitiva, foram elaborados métodos de análise destas verbalizações que levam em conta o grau de diferenciação (individuação) e de relacionamento, a organização cognitiva e dimensões qualitativas na descrição do self e do objeto (Diamond et al., 1991).

Duas dimensões fundamentais do self e da representação do objeto foram consideradas, baseadas na noção de sepa- ração e individuação de Mahler (1993), as quais incluem a representação do objeto como separado do self, possuindo estados internos próprios, e a constância da representação de si mesmo, relativa a um sentido de identidade pessoal. Desta forma, foram levados em consideração para avaliação das descrições os aspectos relativos a representação mental do objeto, incluindo a capacidade de constância de limites na fronteira entre o self e o objeto, a constância emocional, relativa ao vínculo afetivo, a constância objetal, associada à permanência do objeto, a constância do self, caracterizando uma representação de si mesmo consolidada, e o pensamento operacional, relativo à capacidade de abstração. Assim, a noção de um sentido de self e o desenvolvimento de formas maduras de relação com os demais fundamentaram a organização do Inventário das Relações Objetais (ORI) (Blatt \& Auerbach, 2003), instrumento que é formado por três escalas de avaliação. As escalas podem ser avaliadas em termos qualitativos e intepretativos e, também, podem ser gerados escores das categorias conforme o nível de classificação (ver Tabelas 1 e 2).

A primeira, Escala de Diferenciação-Relacionamento (Diamond et al., 1991) avalia qualitativamente, a partir das verbalizações livres do sujeito, a capacidade representacional do self e do objeto. Estas representações podem ser classificadas em 10 categorias, que vão desde um nível de diferenciação precário, ou até inexistente, até o nível onde a capacidade de representação de si mesmo e dos demais é totalmente diferenciada, recíproca e contínua (Tabela 1). Nas primeiras categorias, associadas a estados mais regressivos e primitivos, tais como em psicopatologias graves, o objeto e o sujeito são descritos de forma fusionada, sem diferenciação entre os estados internos do self e do outro. Uma descrição nesta categoria incluiria narrativas descrevendo a pessoa significativa, como: "Somos um só, o que eu sinto ele(a) sente. Tenho medo quando me olha, porque ele(a) sabe o que estou pensando". Por sua vez, as categorias intermediárias incluem esforços no sentido da diferenciação, ocorrendo descrições concretas dos demais em termos de atributos físicos ou funções, porém com pouco reconhecimento de seus estados internos. Neste caso, as narrativas se embasariam em descrições como: "Ele(a) é alto, forte, trabalha, mas passa o tempo todo me incomodando. Tudo o que eu quero, ele(a) critica." Já nas categorias superiores, as descrições discriminam as características de forma mais precisa, incluindo tanto a diferenciação do self em relação aos outros quanto interpretações sobre os estados internos dos demais e suas intenções, portanto baseadas em perspectivas complexas de análise. Nesta categoria, ocorre um esforço maior de tentar compreender diferentes aspectos do objeto: "Ele(a) é alto, forte, trabalha. Às vezes sinto que me critica bastante, mas creio que isso resultou da educação que teve. Os pais sempre exigiram muito dele. É difícil para eu aceitar, mas tento ver o lado dele(a)."

Portanto, a análise das descrições leva em conta o nível de diferenciação identificado através das categorias. Por exemplo, em uma investigação sobre a mudança terapêutica em pacientes com diagnósticos graves, Gruen e Blatt (1990) observaram mudanças na representação mental do self e dos demais ao longo do tratamento. Estes pacientes foram avaliados ao iniciar o atendimento e, posteriormente, aos 6 meses e, 
assim, sucessivamente. A partir da relação transferencial, os autores verificaram movimentos em direção a representações mais coesas, ainda que particulares a cada caso, considerando características afetivas e conflitivas associadas aos quadros esquizofrênicos e borderlines. Assim, iniciando com descrições concretas do pai, mãe e de si mesmos, que incluíam pouca diferenciação entre o sujeito e objeto, os pacientes passaram gradualmente a incluir descrições um pouco mais precisas e diferenciadas.

Para complementar a análise das descrições, a segunda Escala do Nível Conceptual avalia a estrutura descritiva do self e do objeto e é classificada nos seguintes níveis: sensório-motor, perceptual concreto, icônico externo, icônico interno, sendo o último, o nível conceptual de representação (Tabela 2). No primeiro nível, sensório-motor, a pessoa é descrita em termos da gratificação ou frustração que pode causar ao sujeito. Assim, a perspectiva de análise é sempre em termos das necessidades do sujeito. No nível concreto, a pessoa é descrita em termos de qualidades ou características reais. No icônico externo, a descrição considera as funções ou atividades funcionais da pessoa. E no icônico interno, suas opiniões e pensamentos. No último nível, o self e o objeto são descritos em uma riqueza de significados, incluindo tanto aspectos concretos como qualidades, opiniões, sentimentos e idéias. Todos os aspectos incluem pontos contraditórios e ambivalentes, que são integrados em um todo único e singular.
Finalmente, a Análise Temática das verbalizações permite a classificação de adjetivos das descrições de si e dos demais em termos da qualidade associada às descrições. Incluem, por exemplo, dimensões como, afetividade, ambição, características malevolentes ou benevolentes, frio-carinhoso, envolvimento construtivo, características intelectuais, ideais positivos e negativos, punição, sucesso e fraqueza.

A integração destes três níveis de avaliação permite que se identifiquem os diferentes elementos constitutivos das representações do self e do objeto, os quais refletirão as características do funcionamento psíquico do sujeito, incluindo desde representações associadas a estruturas neuróticas, psicóticas ou borderline. Considera-se que no nível psicótico há uma dificuldade de manutenção das fronteiras do self e objeto, enquanto no nível borderline, mantêm-se essas fronteiras, mas há uma significativa ambivalência afetiva em relação ao objeto. Finalmente, o neurótico, constitui-se em descrições mais elaboradas, coesas, ocorrendo tentativas de síntese que são prejudicadas em função dos conflitos nessas relações. $\mathrm{O}$ estudo destas representações no processo clínico torna-se, portanto, um elemento importante no diagnóstico e no acompanhamento do trabalho terapêutico.

Estudos utilizando o Inventário de Relações Objetais identificaram mudanças nos níveis descritivos de pacientes em psicoterapia de orientação psicanalítica, indicando que o processo terapêutico possibilita que as representações

Tabela 1. Diferenciação - Relação entre o Self e a Representação Objetal.

Categorias do self Descrição

Comprometimento no limite com o objeto.

Confusão no limite com o objeto.

Espelhamento no limite com o objeto.

Idealização ou desvalorização objetal.

Semi-diferenciação.

Emergência de constância ambivalente do self.

Consolidação e constância - perspectiva unilateral.

Coesão, individuação.

Coesão, relação recíproca, integrada.

Coesão, perspectiva complexa das interações.
Senso básico de coesão física e/ou integridade representacional são inexistentes.

Self e os demais representados fisicamente intactos, mas sentimentos e pensamentos são amorfos e indiferenciados.

Características do self e do objeto-aparência, qualidades corporais, forma e tamanho são virtualmente idênticas.

Tentativas de consolidar a representação baseada em idealização ou ataque. Pontos de vista extremados.

Marcadas oscilações entre opostos de qualidades ou aspectos concretos (polarização em partes concretas).

Consolidação de aspectos divergentes do self e do objeto, ainda hesitantes, ambivalentes e sem singularidade. Tentativa de representação mais individuada e coesa.

Pensamentos, sentimentos, necessidades são diferenciadas e moduladas. Maior integração de aspectos contraditórios. Compreensão simpática dos demais.

Coesão e sentido de relação com os demais. Definição de identidade, compreensão da perspectiva dos demais.

Senso coeso de self e do objeto em relações que modificam a ambos de forma significativa.

Relações recíprocas e integradas, perspectiva complexa, clara compreensão dos aspectos interpessoais influenciando as relações. 
Tabela 2. Escala Nível Conceptual das Descrições do Self e das Representações Objetais.

\begin{tabular}{ll}
\hline Nível & Descrição \\
\hline $\begin{array}{l}\text { 1. Sensório motor-Pré operacional } \\
\text { 2. Perceptual-Concreto }\end{array}$ & $\begin{array}{l}\text { A pessoa é descrita em termos da gratificação ou frustração que ela proporciona ao sujeito. Não } \\
\text { há experiência da pessoa como separada do sujeito. }\end{array}$ \\
$\begin{array}{l}\text { 3. Icônico } \\
\text { Icônico Externo }\end{array}$ & $\begin{array}{l}\text { Características físicas e atividades do objeto restritas, sem dimensão interna, integração de } \\
\text { pontos conflitivos, centradas em aspectos extremos do objeto. }\end{array}$ \\
Icônico Interno & $\begin{array}{l}\text { Maior ênfase nos atributos, pensamentos, sentimentos do sujeito descrito - dimensões psicológi- } \\
\text { cas internas. }\end{array}$ \\
A descrição inclui uma vasta gama de dimensões, desde características físicas, atividades, pen- \\
samentos, aspectos contraditórios e complexos. Estes são integrados de forma coerente.
\end{tabular}

Adaptado de Blatt \& Auerbach (2003).

mentais organizem-se ao longo do trabalho de forma mais diferenciada e, desta forma, organizando as experiências afetivas do sujeito (Blatt \& Auerbach, 2003). Ao nível de estudos em psicopatologia, em uma investigação com adolescentes, Besser e Blatt (2007) verificaram que o nível conceitual das representações objetais diferenciava-se em relação às patologias internalizantes e externalizantes. Isto é, nos casos de adolescentes depressivos a representação paterna era a que atingiu o nível mais alto de descrição e, ao contrário, nos adolescentes com indicadores agressivos as representações mais altas foram as das mães. Estas variações sugerem caminhos interessantes de pesquisa sobre o processo de construção de identidade na adolescência.

Observa-se, a partir destes trabalhos que a análise das transformações psíquicas associadas às características das representações mentais constitui-se como uma base importante para o trabalho clínico. E, da mesma forma, fornecem elementos de análise tanto para a compreensão das patologias como para o acompanhamento do processo terapêutico.

\section{Considerações Finais}

Neste trabalho foram apresentadas e discutidas as implicações teóricas do conceito de representação mental, a partir dos enfoques da teoria das relações objetais e teoria do apego, destacando-se também os aspectos referentes à compreensão e avaliação da mudança terapêutica no tratamento psicanalítico. Especificamente, com base na contribuição de Blatt acerca da possibilidade de transformação dessas representações no encontro terapêutico, foram apontadas perspectivas interessantes de estudos sobre a mudança terapêutica.

Além disso, considerando-se a ênfase das abordagens psicanalíticas nos processos de mudança das estruturas mentais e dos estados inconscientes como as principais metas terapêuticas, a utilização do instrumento de avaliação das representações mentais ORI permite precisamente identificar esses propósitos. Portanto, a importância de aprofundar a definição e métodos de análise da representação mental reside não somente na possibilidade de aprimorar o trabalho clínico em psicoterapias como também de permitir o desenvolvimento de pesquisas em psicanálise através de estudos voltados para maior compreensão do processo terapêutico. Desta forma, fundamentando-se em constructos consistentes será possível o desenvolvimento e ampliação da pesquisa psicanalítica de forma a contribuir no campo da psicopatologia e da intervenção.

Em suma, a mudança terapêutica pode ser compreendida e avaliada sob diferentes enfoques. Todavia, a perspectiva da representação mental do objeto na avaliação do trabalho clínico possibilita um melhor entendimento da influência mutativa do trabalho psicanalítico no processo de reorganização e integração das estruturas mentais e do estado afetivo do sujeito. Desta maneira, este constructo torna-se um elemento promissor para a investigação e sistematização do processo terapêutico na abordagem psicanalítica, permitindo a identificação dos diferentes elementos associados à mudança.

\section{Referências}

Ainsworth, M. D. S. (1969). Object relationships, dependency, and attachment: A theoretical review of the infant-mother relationship. Child Development, 40, 969-1026.

Barish, K. (2004). What is therapeutic in child therapy? I. Therapeutic engagement. Psychoanalytic Psychology, 21, 385-401.

Beres, M. D., \& Joseph, E. D. (1970). Beres, D., Joseph, E.D. (1970). The concept of mental representation in psychoanalysis. International Journal of Psychoanalysis, 51,1-8.

Berti, T. R. (2004). Mudança psíquica: Uma revisão. Revista Brasileira de Psicoterapia, 1, 35-49.

Besser, A., \& Blatt, S. J. (2007). Identity consolidation and internalizing and externalizing problem behaviors in early adolescence. Psychoanalytic Psychology, 24, 125-149.

Blatt, S. J., Auerbach, J. S., Zuroff, D. C., \& Shahar, G. (2006). Evaluating efficacy, effectiveness, and mutative factors in psychodynamic psychotherapies. In PDM Task Force. Psychodynamic diagnostic manual (pp. 537-572). Silver Spring, MD: Alliance of Psychoanalytic Organizations. 
Blatt, S., \& Levy, K. N. (2003). Attachment theory, psychoanalysis, personality development, and psychopathology. Psychoanalytic Inquiry, 23, 102-150.

Blatt, S. J., \& Auerbach, J. S. (2003). Psychodynamic measures of therapeutic change. Psychoanalytic Inquiry, 23, 268-307.

Blatt, S. J., Auerbach, J. S., \& Levy, K. N. (1997). Mental representations in personality development, psychopathology, and the therapeutic process. Review of General Psychology, 1, 351-374.

Blatt, S. J., Besser, A., \& Ford, R. Q. (2007). Two primary configurations of psychopathology and change in thought disorder in long-term intensive inpatient treatment of seriously disturbed young adults. American Journal of Psychiatry, 164, 1561-1567.

Blatt, S. J., Chevron, E. S., Quinlan, D. M., Schaffer, C. E., \& Wein, S. (1988). The assessment of qualitative and structural dimensions of object representations. Unpublished research manual. New Haven!: Yale University.

Bowlby, J. (1969). Attachment and loss: Vol. 1: Attachment. New York: Basic Books.

Dalbem, J. \& Dell'Aglio, D. (2006). Teoria do apego: bases conceituais e desenvolvimento dos modelos internos de funcionamento. Arquivos Brasileiros de Psicologia, Retrieved from http://seer.psicologia.ufrj.br/seer/lab19/ojs/viewarticle. php?id=62.

Diamond, D. \& Blatt, S. J. (1994). Internal working models and the representational world in attachment and psychoanalytic theories. In M. B. Sperling \& W. H. Berman (Eds.), Attachment in adults: Clinical and developmental perspective (pp.72-97). New York: The Guilford Press.

Diamond, D., Blatt, S. J., Stayner, D., \& Kaslow, N. (1991). Selfother differentiation of object representations. Unpublished research manual. New Haven!: Yale University.

Fonagy, P., \& Target, M. (1997). Attachment and reflective function: Their role in self-organization. Development and Psychopathology, 9, 679-700.

Fonagy, P., Rotha, A, \& Higgitt, A. (2005). The outcome of psychodynamic psychotherapy for psychological disorders. Clinical Neuroscience Research, 4, 367-377.

Gruen, R. J., \& Blatt, S. J. (1990). Change in self- and object representation during long-term dynamically oriented treatment. Psychoanalytic Psychology, 7, 399-422.

Jung, S. I., Fillippon, A. P. M., Nunes, M. L. T., \& Eizirik, C. L. (2006). História recente e perspectivas atuais da pesquisa de resultados em psicoterapia psicanalítica de longa duração. Revista de Psiquiatria do Rio Grande do Sul, 3, 298-312.
Kernberg, O. F. (2005). Identity diffusion in severe personality disorders. In S. Strack (Ed.), Handbook of personality and psychopathology (pp. 39-49). Hoboken, N. J.: John Wiley $\&$ Sons.

Kernberg, O. F. (2003). Psicanálise, psicoterapia psicanalítica e psicoterapia de apoio: controvérsias contemporâneas. In A. Green (Ed.), Psicanálise contemporânea: Revista Francesa de psicanálise (pp.23-49). Rio de Janeiro: Imago Eds.

Kronmuller, K. T., Postelnicu, I., Hartmann, M., Stefini, A., GiserElze, A., Gerhold, M., Hildegard, H. \& Winkelmann, K. (2005). Efficacy of psychodynamic short-term psychotherapy for children and adolescents with anxiety disorders. Praxis der Kinderpsychologie und Kinderpsychiatrie, 54, 559-577.

Mahler, M. S. (1993). O nascimento psicológico da criança: simbiose e individuação. Porto Alegre: Artes Médicas.

Main, M. (1996). Introduction to the special section on attachment and psychopathology: Overview of the field of attachment. Journal of Consulting and Clinical Psychology, 64, 237-243.

Matos, M. (2004). Acerca da identidade sexual como elemento de ligação somato-psíquica. Revista Portuguesa de Psicossomática, 6, 7-15.

Novey, S. (1958). The meaning of the concept of mental representation of objects. The Psychoanalytic Quarterly, 27, 40-366.

Pine, F. (1998). As quatro psicologias da psicanálise e seu lugar no trabalho clínico. Revista de Psicanálise, 3, 339-359.

Ramires, V. R. R. (2003). Cognição social e teoria do apego: Possíveis articulações. Psicologia: Reflexão e Crítica. Retrieved from http://www.scielo.br/pdf/prc/v16n2/ a20v16n2.pdf.

Russell, R. L., \& Orlinsky, D. E. (1996). Psychotherapy research in historical perspective: implications for mental health care policy. Archives of General Psychiatry, 53, 708-715.

Zaslavsky, J. (2005). Por que publicar a pesquisa qualitativa em psicoterapia e psicanálise? (Editorial). Revista de Psiquiatria do Rio Grande do Sul, 3, 238-239.

Recebido em 03.10.2009

Primeira decisão editorial em 17.10.2010

Versão final em 24.10.2011

Aceito em 10.11.2011 